\title{
Analysis of the Critical Success Factors of SOA Implementation in China Tobacco Company Based on DEMATEL Approach
}

\author{
Yong Cen \\ Information Center, China Tobacco Zhejiang Industrial Co., Ltd \\ Hangzhou, 310009, China
}

Keywords: service oriented architecture, critical success factors, DEMATEL, SOA implementation.

\begin{abstract}
Despite the widespread use of SOA design principles, it remains not only technically difficult to implement, but also presents a substantial challenge to systems architects and managers. This study provides a methodology to evaluate SOA implementation critical success factors (CSFs) that can aid managers make proper SOA investment strategies. Through a comprehensive systematic review of the SOA literature and 15 SOA-specific CSFs for the successful implementation of SOA are identified. This paper use DEMATEL approach to visualize the structure of complicated causal relationships between these CSFs and obtain the influence level of these factors. The five most important factors found in the study, from amongst the identified CSFs; include deepening of enterprise-wide perception of SOA, long-term planning and step-by-step evolution planning with consideration of current capacity, Enterprise-wide management support, project team and standardization of business process. We also found a number of direct and indirect relationships amongst the CSF factors.
\end{abstract}

\section{Introduction}

At present, service oriented architecture (SOA) is one of the most popular topics which is an architecture building method used to describe, link and integrate the reusable business services with clear boundary and self-contained functions. The paradigm of SOA has recently emerged as a conceptual architecture framework facilitating this change in allowing agile business processes to adjust flexibly to a changing environment [1]. This shift in paradigm towards SOA is intended to meet firms' needs to respond quickly to rapidly changing market demands. In fact, the dynamic capability of a firm to adapt to changes in both its internal and external environment has become an aspect of its core competency [2]. Organizations that implement SOA can: reduce costs, reuse software (e.g. web services), integrate their legacy systems and IT infrastructures, and better align business with IT [3].

However, based on statistics in Heffner's reports, it is obvious that 58 percent of SOA adopters have not yet figured out how to benefit from their projects [4]. Thus, even though SOA is considered as a valuable architectural paradigm, there exist factors that are unclear to implementers exist. These are mainly non-technical factors and can affect the success of SOA implementations [5, 6].

This paper therefore aims to determine the CSFs for successful SOA implementation in China Tobacco Company and to describe the practices that lead to the presence of these factors. Through an intensive literature review, this study has managed to extract influencing factors from the summary of criteria in former research. Thus, a decision-making trial and evaluation laboratory (DEMATEL) method [7] is adapted to construct a network structure of interdependent factors. Using this method, could gather group knowledge to form a structural model, and then visualize the causal relationship among factors through a cause-effect relationship diagram. The outcomes of DEMATEL provide information about the impact each factor has on the SOA implementation. Through analysing and discussing the structural model, we can figure out which factors are of more fundamental importance for the whole implementation process, and which are not. 


\section{SOA implementation critical success factors: a literature review}

SOA is an architectural paradigm in which business processes are composed using web services. From a technical perspective, SOA addresses issues related to reuse, maintenance and integration. From a business perspective, SOA provides services that are aligned with internal or external business needs. SOA provides an opportunity to achieve broad-scale interoperability, while offering flexibility to adapt to changing technologies and business requirements [8].

Despite the benefits that SOA provides, not all SOA projects have delivered the expected outcomes due to organizations lack of understanding especially in non-technical factors (managerial, human, etc.) surrounding SOA implementations [4]. For this reason, we decided to focus our study on CSFs of SOA implementations in China Tobacco Company given that: SOA projects are affected by various factors and the importance of projects success. Yoon and Carter investigate the antecedents and benefits of SOA implementation through five different case studies, stressing the importance of IT and business alignment besides other critical factors such as effective governance, senior management, and SOA registries [9]. Biemborn et al. given a conceptual research model suggests that system compatibility and complexity may drive SOA business value, while a discrepancy between business needs and SOA system features may inhibit its successful use [10].

Konstantinos et al. explored numerous factors affect SOA success through literature review, then analysed and identify critical success factors influencing SOA implementation in healthcare [6]. Aier et al. studied focus on what are the characteristics of successful implementations of service oriented information systems, and what are the critical success factors influencing driving and/or, determining these characteristics through empirical analysis [11]. Owens et al. conducted a comprehensive systematic review of the SOA literature, and identified five SOA-specific CSFs may be critical for realizing the benefits of SOA [12]. Lee et al. takes the form of an exploratory study based on a review of SOA literatures and interviews and claims to identify twenty factors in successful SOA adoption, and subsequently defines a concrete implementation policy for each factor [13]. Haresh and Fethi reviewed the current state of the technology, identified the factors influencing the decision to adopt service-oriented computing as an enterprise strategy and discussed the associated research literature, and concluded with a suggested research agenda and conceptual framework for investigating the use of service-oriented computing in practice [14]. Based on the 15 individual factors that are derived from the literature as follow the table 1 shown:

Table 1: Summary of CSFs

\begin{tabular}{ll}
\hline ID & \multicolumn{1}{c}{ Critical Success Factors } \\
\hline X1 & Deepening of enterprise-wide perception of SOA \\
X2 & Enterprise-wide management support \\
X3 & Clear goal-setting based on business value \\
X4 & Long-term planning and step-by-step evolution planning \\
& with consideration of current capacity \\
X5 & Framing an organizational model for SOA management \\
X6 & Fostering a partnership culture between business and IT \\
X7 & Generating standard definitions of SOA technology \\
X8 & Defining scope of technology application / security \\
& foundation \\
X9 & Standardization of business process \\
X10 & Definition of SOA-based development methodology \\
X11 & Project team \\
X12 & Strengthening business service-oriented design process \\
X13 & Managing SOA policy processes \\
X14 & Establishing a service development/ operation management \\
& process \\
X15 & Risks management \\
\hline
\end{tabular}




\section{DEMATEL methodology}

DEMATEL is built on the basis of graph theory, enabling analyses and solves problems by visualization method. This structural modelling approach adopts the form of a directed graph, a causal-effect diagram, to present the interdependence relationships and the values of influential effect between factors. Through analysis of visual relationship of levels among system factors, all elements are divided into causal group and effected group. And this can help researchers better understand the structural relationship between system elements, and find ways to solve complicate system problems $[15,16]$. The procedure of DEMATEL method can be depicted by the following five steps:

Step1: Generate the initial direct-relation matrix. Form a committee of experts, and acquire the assessments about direct affect between each pair of elements. Converting the linguistic assessments into crisp values, we obtain the direct-relation matrix $X=\left[x_{i j}\right]$, where is $X$ a $n \times n$ nonnegative matrix, $x_{i j}$ indicates the direct impact of factor $i$ on factor $j$.

Step2: Normalize the initial direct-relation matrix. The normalized direct-relation matrix $D=\left[d_{i j}\right]$ can be obtained through Eq. (1).

$$
D=\frac{1}{\max _{1 \leq i \leq n} \sum_{j=1}^{n} x_{i j}} X
$$

Step3: Acquire the total-relation matrix $T$ using the Eq. (2) in which $I$ is an $n \times n$ identity matrix. The element $t_{i j}$ indicates the indirect effects that factor $i$ on factor $j$, so the matrix can reflect the total relationship between each pair of system factors.

$$
T=D(I-D)^{-1}
$$

Step4: Calculate the sum of rows and columns of matrix $T$. To make the outcome more visible, we compute $D_{i}$ and $R_{j}$ through Eq. (3) and (4), respectively. The sum of row $i$, which is denoted as $D_{i}$, represents all direct and indirect influence given by factor to all other factors, and so $D_{i}$ can be called the degree of influential impact. Similarly, the sum of column $j$, which is denoted as $R_{j}$ can be called as the degree of influenced impact, since $R_{j}$ summarizes both direct and indirect impact received by factor $j$ from all other factors.

$$
\begin{aligned}
D_{i} & =\sum_{1 \leq j \leq n} x_{i j} \\
R_{j} & =\sum_{1 \leq i \leq n} x_{i j}
\end{aligned}
$$

Step5: Construct cause-effect relationship diagram based on $D_{i}+R_{i}$ and $D_{i}-R_{i}$. A cause-effect diagram can be drawn by mapping the dataset of $\left(D_{i}+R_{i}, D_{i}-R_{i}\right)$. And the complex interrelationship among factors is visualized through the diagram construction process.

\section{Critical success factor analysis of SOA implementation}

Application of proposed method.The critical success factors influencing SOA implementation are analyzed and structured following the proposed method. In the first step a questionnaire was issued to 7 respondents (managers, IT staffs, consultants), and they were asked to evaluate the direct influence between any two factors in those illustrate 15 CSFs (1-least important, 2-less important, 3-important, 4-very important, and 5-crucial). Thus, the normalized initial direct-relation matrix as Table 2 is obtained. Next using Eq. (2), the total-relation matrix (Table 3) is calculated. So indexes and scores of each factor can be obtained using Eq. (3) and (4), as shown in Table 4. 
Table 2: The normalized initial direct-relation matrix

\begin{tabular}{|c|c|c|c|c|c|c|c|c|c|c|c|c|c|c|c|}
\hline ID & $\mathrm{X} 1$ & $\mathrm{X} 2$ & $\mathrm{X} 3$ & $\mathrm{X} 4$ & X5 & X6 & $\mathrm{X} 7$ & $\mathrm{X} 8$ & X9 & $\mathrm{X} 10$ & X11 & $\mathrm{X} 12$ & $\mathrm{X} 13$ & X14 & X15 \\
\hline $\mathrm{X} 1$ & 0.0000 & 0.0784 & 0.0784 & 0.0784 & 0.0784 & 0.0588 & 0.0196 & 0.0196 & 0.0588 & 0.0392 & 0.0784 & 0.0196 & 0.0588 & 0.0392 & 0.0784 \\
\hline $\mathrm{X} 2$ & 0.0588 & 0.0000 & 0.0784 & 0.098 & 0.0784 & 0.0784 & 0.0392 & 0.0392 & 0.0784 & 0.0392 & 0.0784 & 0.0392 & 0.0784 & 0.0588 & 0.0784 \\
\hline X3 & 0.0588 & 0.0784 & 0.0000 & 0.0784 & 0.0588 & 0.0588 & 0.0392 & 0.0392 & 0.0588 & 0.0392 & 0.0588 & 0.0392 & 0.0588 & 0.0392 & 0.0588 \\
\hline $\mathrm{X} 4$ & 0.0392 & 0.0784 & 0.098 & 0.0000 & 0.0784 & 0.0784 & 0.0588 & 0.0784 & 0.0784 & 0.0588 & 0.0784 & 0.0392 & 0.0784 & 0.0392 & 0.0784 \\
\hline X5 & 0.0588 & 0.0588 & 0.0588 & 0.0588 & 0.0000 & 0.0784 & 0.0588 & 0.0588 & 0.0588 & 0.0588 & 0.0784 & 0.0588 & 0.0588 & 0.0392 & 0.0588 \\
\hline X6 & 0.0392 & 0.0588 & 0.0392 & 0.0784 & 0.0588 & 0.0000 & 0.0392 & 0.0392 & 0.0784 & 0.0392 & 0.0588 & 0.0392 & 0.0784 & 0.0588 & 0.0784 \\
\hline X7 & 0.0196 & 0.0196 & 0.0196 & 0.0196 & 0.0196 & 0.0196 & 0.0000 & 0.098 & 0.0784 & 0.0784 & 0.0196 & 0.0784 & 0.0784 & 0.0784 & 0.0392 \\
\hline X8 & 0.0392 & 0.0196 & 0.0196 & 0.0392 & 0.0588 & 0.0196 & 0.0784 & 0.0000 & 0.0784 & 0.0784 & 0.0196 & 0.098 & 0.0784 & 0.098 & 0.0588 \\
\hline X9 & 0.0196 & 0.0588 & 0.0588 & 0.0588 & 0.0588 & 0.0392 & 0.0588 & 0.0784 & 0.0000 & 0.0784 & 0.0392 & 0.0588 & 0.0392 & 0.0588 & 0.0588 \\
\hline X10 & 0.0196 & 0.0196 & 0.0196 & 0.0392 & 0.0392 & 0.0392 & 0.0784 & 0.0588 & 0.0784 & 0.0000 & 0.0392 & 0.0784 & 0.0588 & 0.0784 & 0.0588 \\
\hline X11 & 0.0588 & 0.0784 & 0.0784 & 0.0784 & 0.0784 & 0.0784 & 0.0588 & 0.0588 & 0.0784 & 0.0588 & 0.0000 & 0.0588 & 0.0784 & 0.0784 & 0.0784 \\
\hline $\mathrm{X} 12$ & 0.0392 & 0.0196 & 0.0392 & 0.0196 & 0.0588 & 0.0392 & 0.0784 & 0.0784 & 0.0588 & 0.0784 & 0.0392 & 0.0000 & 0.0588 & 0.0784 & 0.0588 \\
\hline $\mathrm{X} 13$ & 0.0196 & 0.0196 & 0.0392 & 0.0196 & 0.0392 & 0.0392 & 0.0588 & 0.0588 & 0.0392 & 0.0588 & 0.0392 & 0.0588 & 0.0000 & 0.0784 & 0.0588 \\
\hline X14 & 0.0392 & 0.0392 & 0.0196 & 0.0392 & 0.0392 & 0.0196 & 0.0392 & 0.0588 & 0.0588 & 0.0588 & 0.0196 & 0.0588 & 0.0588 & 0.0000 & 0.0588 \\
\hline $\mathrm{X} 15$ & 0.0196 & 0.0392 & 0.0588 & 0.0588 & 0.0588 & 0.0196 & 0.0392 & 0.0392 & 0.0392 & 0.0196 & 0.0784 & 0.0196 & 0.0392 & 0.0196 & 0.0000 \\
\hline
\end{tabular}

Table 3: The total-relation matrix

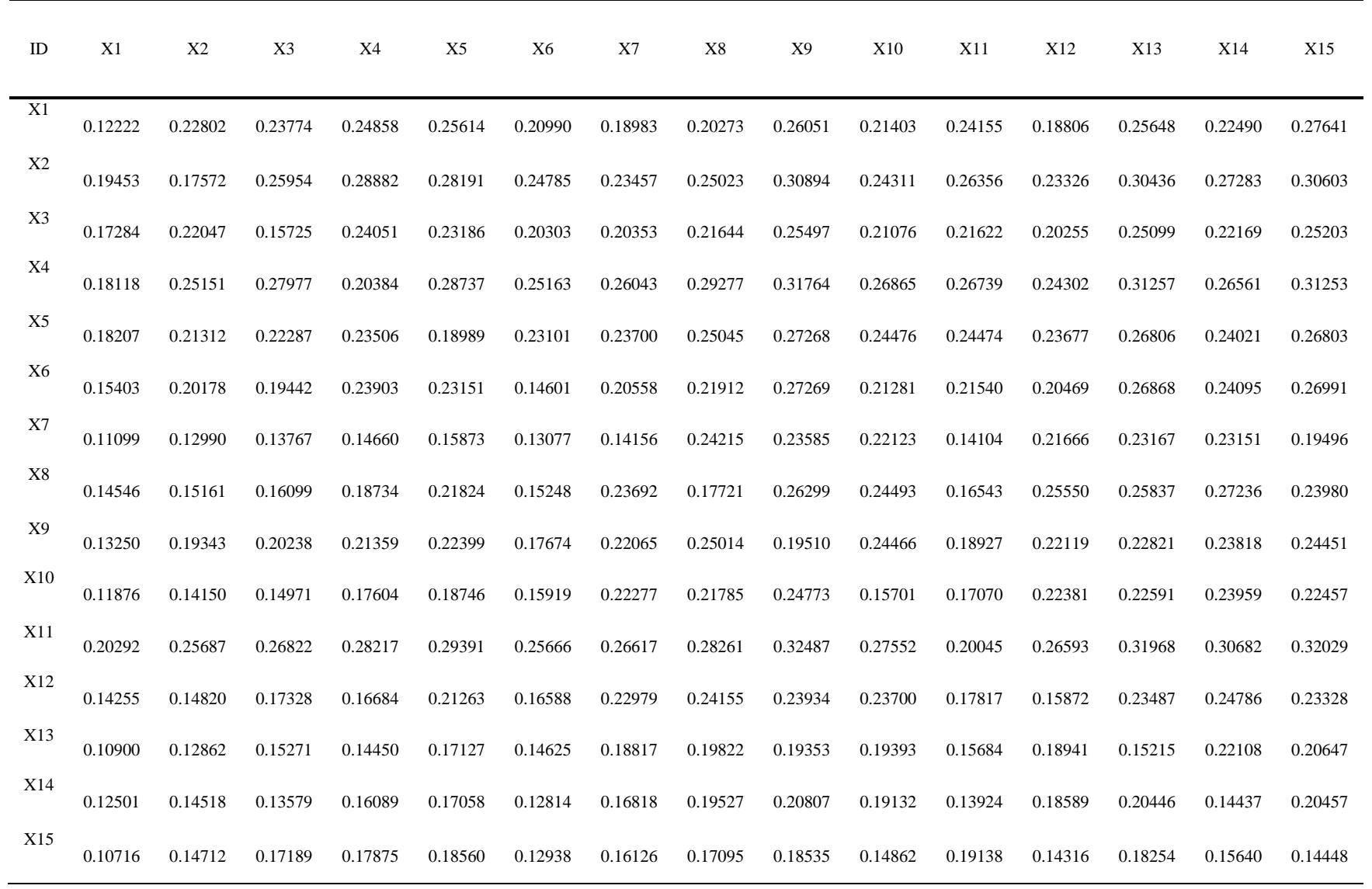


Table 4: The scores of each factor and related values for cause and effect groups

\begin{tabular}{|c|c|c|c|c|c|}
\hline ID & CSFs & $D_{i}$ & $\overline{R_{j}}$ & $D_{i}+R_{i}$ & $D_{i}-R_{i}$ \\
\hline $\mathrm{X1}$ & $\begin{array}{l}\text { Deepening of enterprise-wide perception of } \\
\text { SOA }\end{array}$ & 3.3571 & 2.20120 & 5.55830 & 1.15590 \\
\hline $\mathrm{X} 2$ & Project team & 3.8653 & 2.733 & 6.59830 & 1.13230 \\
\hline X3 & Clear goal-setting based on business value & 3.2551 & 2.9042 & 6.15930 & 0.35090 \\
\hline $\mathrm{X} 4$ & $\begin{array}{l}\text { Long-term planning and step-by-step } \\
\text { evolution planning with consideration of } \\
\text { current capacity }\end{array}$ & 3.9959 & 3.1126 & 7.10850 & 0.88330 \\
\hline X5 & $\begin{array}{c}\text { Framing an organizational model for SOA } \\
\text { management }\end{array}$ & 3.5367 & 3.3011 & 6.83780 & 0.23560 \\
\hline X6 & $\begin{array}{c}\text { Fostering a partnership culture between } \\
\text { business and IT }\end{array}$ & 3.2766 & 2.7349 & 6.01150 & 0.54170 \\
\hline $\mathbf{X 7}$ & $\begin{array}{c}\text { Generating standard definitions of SOA } \\
\text { technology }\end{array}$ & 2.6713 & 3.1664 & 5.83770 & -0.49510 \\
\hline $\mathbf{X 8}$ & $\begin{array}{l}\text { Defining scope of technology application / } \\
\text { security foundation }\end{array}$ & 3.1296 & 3.4077 & 6.53730 & -0.27810 \\
\hline X9 & Standardization of business process & 3.1745 & 3.7802 & 6.95470 & -0.60570 \\
\hline X10 & $\begin{array}{c}\text { Definition of SOA-based development } \\
\text { methodology }\end{array}$ & 2.8626 & 3.3083 & 6.17090 & -0.44570 \\
\hline X11 & Enterprise-wide management support & 4.1231 & 2.9814 & 7.10450 & 1.14170 \\
\hline X12 & $\begin{array}{l}\text { Strengthening business service-oriented } \\
\text { design process }\end{array}$ & 3.01 & 3.1686 & 6.17860 & -0.15860 \\
\hline X13 & Managing SOA policy processes & 2.5521 & 3.699 & 6.25110 & -1.14690 \\
\hline X14 & $\begin{array}{l}\text { Establishing a service development/ } \\
\text { operation management process }\end{array}$ & 2.507 & 3.5244 & 6.03140 & -1.01740 \\
\hline $\mathbf{X 1 5}$ & Risks management & 2.404 & 3.6979 & 6.10190 & -1.29390 \\
\hline
\end{tabular}

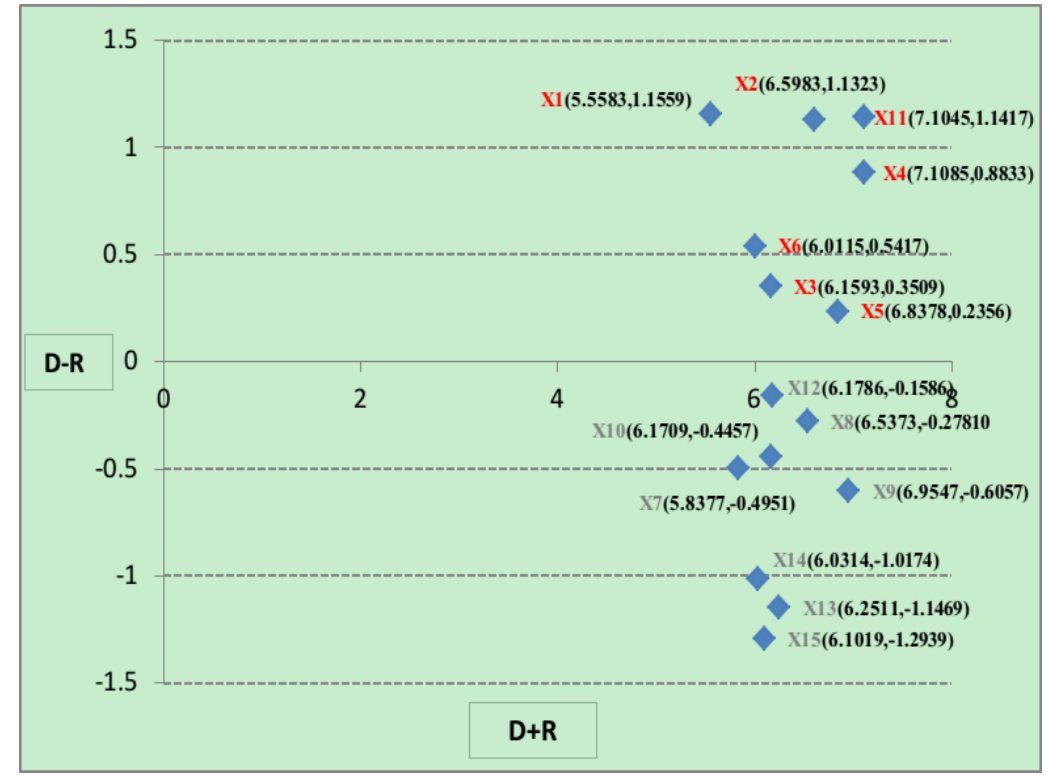

Fig.1: The cause-effect relationship diagram

According to the data in Table 3 and Table 4, the cause-effect relationship diagram is acquired by mapping the dataset of $\left(D_{i}+R_{i}, D_{i}-R_{i}\right)$. As shown in Fig. 1, the factors are visually divided into two groups according to whether its value of $D_{i}-R_{i}$ is positive or negative. So the cause group with positive $D_{i}-R_{i}$ value includes X1, X2, X3, X4, X5, X6, X11 and other factors including X7, X8, X9, $\mathrm{X} 10, \mathrm{X} 12, \mathrm{X} 13, \mathrm{X} 14, \mathrm{X} 15$ are in the effect group since the $D_{i}-R_{i}$ of these factors are negative. There are many other valuable clues that can be obtained from Fig. 1 to facilitate to make decisions. 
Indeed $D_{i}+R_{i}$ is the prominence and $D_{i}-R_{i}$ is the relationship. That is, $D_{i}+R_{i}$ illustrates the importance of the factor (the strength of both given and received influences of this factor to and from other factors). In general when $D_{i}-R_{i}$ is positive the factor is a part of the cause group and when it is negative, the factor is of the affected group.

\section{Analysis of critical success factors.}

\section{1)Cause factors analysis}

Among all factors in cause group, X11 (Enterprise-wide management support) has the second place $D_{i}-R_{i}$, which means that X11 dispatches remarkable impact on the whole implementation than that it receives from other factors. Besides, Table 4 shows that the degree of influential impact of X11 is 4.1231, which ranks first place among all causal factors. It is indicated that X11 has more impact on other factors, and that improvement of X11 can lead to the success of the SOA implementation and worth much more attention.

The factor having the first highest $D_{i}-R_{i}$ is factor X1(Deepening of enterprise-wide perception of SOA). However, as shown in Fig. 1 , its score in the degree of importance $D_{i}+R_{i}$ is relatively very low. We further analyze the indexes in Table 4 and find out the reason for it. According to the $D_{i}$ and $R_{i}$ scores of $\mathrm{X} 1$, its influential impact on others is high while the impact it receives from others is the most small, which leads to a small value of $D_{i}+R_{i}$. Nevertheless, this relatively small value of $D_{i}+R_{i}$ could not dispute the fact that factor $\mathrm{X} 1$ has a great impact on the whole implementation. Meanwhile, X2 (Project team) is for similar reasons. So, X1 and X2 can be clustered as a CSF.

The factor X4 (Long-term planning and step-by-step evolution planning with consideration of current capacity) and X5(Framing an organizational model for SOA management), the value of $D_{i}-R_{i}$ is not high, but its score in the degree of importance $D_{i}$ and $D_{i}+R_{i}$ are very high, thus, the fact that factor X4 and X5 have a great impact on the whole implementation.

According to the outcomes derived from DEMATEL method, the impact dispatched from X3(Clear goal-setting based on business value) and X6(Fostering a partnership culture between business and IT)to other factors is greater than the impact it receives. But both the $D_{i}$ and $R_{i}$ scores of X3 and X6 are not high enough. It is obvious that the two factors do not have notable impact on the whole implementation.

\section{2)Effect factors analysis}

Generally speaking, factors in effect group are tend to be easily impacted by others, which makes effect factors unsuitable to be a critical success factor. Nevertheless, it is still necessary to discuss effect factors to find out the feature of these factor.

Among all eight effect factors, X9(Standardization of business process) has the highest $D_{i}+R_{i}$, showing that it is of the most importance for the operation. But in Fig. 1 we can see that the $D_{i}-R_{i}$ score of X9 is -0.60570 , a value slightly less than zero announcing X9 as a net effect factor. To further illustrate this phenomenon, its degree of influenced impact $R_{i}$ which is 6.9547, ranks the third highest among all system factors, meanwhile, its degree of influential impact index $D_{i}$ is high. This suggests that although X9 is a net receiver, it has an apparent impact on other factors and on the implementation. So considering the important position of $\mathrm{X} 9$ in the SOA implementation.

From the cause-effect relationship diagram, we can see that factor X8 (Defining scope of technology application / security foundation) is an effect factor with $D_{i}-R_{i}$ as -0.2781 slightly below zero. It suggests that X8 is just slightly net affected by other system factors, and it also has a considerable impact on the implementation at the same time. But Table 4 reveals that the importance degree $D_{i}+R_{i}$ of X8 is 6.5373, a number not high enough to label it as a CSF. Besides, the factor $\mathrm{X} 8$ is more independent and there are fewer factors which will impact this variable.

There are some features in common of the rest factors including X7(Generating standard 
definitions of SOA technology), X10(Definition of SOA-based development methodology), $\mathrm{X} 12$ (Strengthening business service-oriented design process), X13(Managing SOA policy processes), X14(Establishing a service development/ operation management process) and X15(Risks management). They are all net effect factors, their importance values $D_{i}+R_{i}$ are low, and their $D_{i}-R_{i}$ are very low scores of negative value suggesting a strong influenced degree. Therefore, all these factors can be easily improved by adjusting other factors, and thus cannot have critical impact on the implementation to promote the success of the SOA implementation.

\section{Conclusions}

In this paper used DEMATEL method to figure out CSFs in accordance with potentially numerous criteria useful for SOA implementation. This method successfully adapts DEMATEL in uncertain situation by applying linguistic variables. This proposed method successfully determined the importance of each factors and further acquired the causal and effect relationships among those 15 factors. Thus, a set of 15 complex influencing factors are divided into a cause group and an effect group, and a visible cause-effect relationship diagram is constructed. In particular, on the basis of cause-effect relationship diagram, five CSFs that are extraordinarily essential for implementation are identified. So the decision makers can apply an implementation approach to ensure the performance of SOA under the constraints of available resources.

Even though some limitations and disadvantages do exist, there is opportunity to investigate how this tool can be used to expand SOA implementation. Not only can DEMATEL be used as a way to handle the interdependencies within a set of criteria, but also can produce more valuable information for making decisions. This method plays an important role in improving SOA implementation strategy and operations especially when it is in a situation where complex environments exist.

\section{References}

[1] Papazoglou, M. P., Georgakopoulos, D. Service-oriented Computing. Communications of the ACM, 46(10), pp. 24-28, 2003.

[2] Sher, P., Lee, V. Information Technology as a Facilitator for Enhancing Dynamic Capabilities through Knowledge Management. Information \& Management, 41 (8), pp. 933-945, 2004.

[3] Mueller Benjamin, G.V., Legner, C. and Riempp, G. Understanding the economic potential of service-oriented architecture. Journal of Management Information Systems, 26(4), pp. 145-180, 2010.

[4] Heffner, R. Forrester Report: Survey Results Show SOAGovernance Improves SOA Benefit Realization. Forrester Inc, Cambridge, MA, 2009.

[5] Morabito, V., Themistocleous, M. and Serrano, A. A survey on integrated IS and competitive advantage. Journal of Enterprise Information Management, 23(2), pp. 201-214, 2010.

[6] Konstantinos Koumaditis, Marinos Themistocleous, Paulo Rupino Da Cunha. SOA implementation critical success factors in healthcare. Journal of Enterprise Information Management, 26(4), pp. 343-362, 2013.

[7] Gabus, A., Fontela, E.. World problems, an invitation to further thought within the framework of DEMATEL. Battelle Geneva Research Centre, Switzerland, Geneva, 1972.

[8] Mahmood, Z. The promise and limitations of service oriented architecture. International Journal of Computers, 1(3), pp. 74-78, 2007.

[9] Yoon, T., Carter, P. (2007). Investigating the Antecedents and Benefits of SOA Implementation: A Multi-Case Study Approach. In AIS (Eds.) Proceedings of the 13th Americas Conference on Information Systems. Colorado, August 9-12. pp. 195-207, 2007. 
[10] Biemborn, D., Joachim, N., Weitzel, T. Drivers and Inhibitors of SOA Business ValueConceptualizing a Research Model. In J. Parsons and Y. Yuan (Eds.) Proceedings of the 14th Americas Conference on Information Systems. Toronto, Canada: Association for Information Systems. pp. 227-238, 2008.

[11] Aier, S., Bucher, T. and Winter, R. Critical success factors of service orientation in information systems engineering. Business \& Information Systems Engineering, 3(2), pp. 77-88, 2011.

[12] Owens, Ian, Cunningham, John. The Identification of Service Oriented Architecture-Specific Critical Success Factors. Proceedings of the 6th European Conference on Information Management and Evaluation. Univ Coll Cork, Cork, IRELAND, SEP 13-14.pp. 267-272, 2012.

[13] Lee, J., Shima, H. and Kim, K. Critical success factors in SOA implementation: an exploratory study. Information Systems Management, 27(2), pp. 123-145, 2010.

[14] Haresh, L., Fethi, R. Service oriented computing in practice - an agenda for research into the factors influencing the organizational adoption of service oriented architectures. Journal of Theoretical and Applied Electronic Commerce Research, 4(1), pp. 256-270, 2009.

[15] Tzeng, G.-H., Chiang, C.-H., and Li, C.-W. Evaluating Intertwined Effects in E-learning Programs: A Novel Hybrid MCDM Model Based on Factor Analysis and DEMATEL. Expert Systems with Applications, 32, pp. 1028-1044, 2007.

[16] Hsin-Hung Wu, Ya-Ning Tsai. An integrated approach of AHP and DEMATEL methods in evaluating the criteria of auto spare parts industry. International Journal of Systems Science, 43(11), pp. 2114-2124, 2012. 